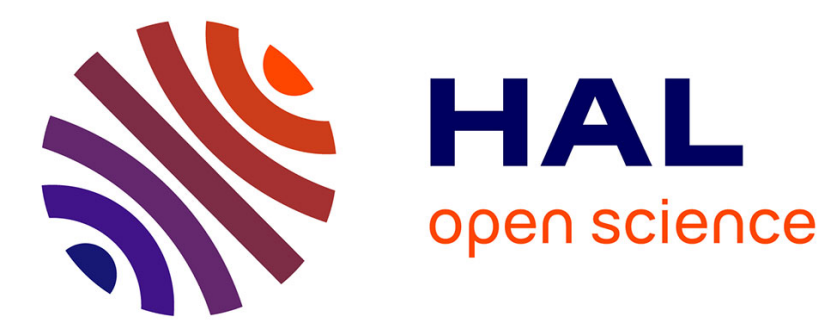

\title{
MAP Estimator for Target Tracking in Wireless Sensor Networks for Unknown Transmit Power
}

Slavisa Tomic, Marko Beko, Rui Dinis, Milan Tuba, Nebojsa Bacanin

\section{To cite this version:}

Slavisa Tomic, Marko Beko, Rui Dinis, Milan Tuba, Nebojsa Bacanin. MAP Estimator for Target Tracking in Wireless Sensor Networks for Unknown Transmit Power. 8th Doctoral Conference on Computing, Electrical and Industrial Systems (DoCEIS), May 2017, Costa de Caparica, Portugal. pp.325-334, 10.1007/978-3-319-56077-9_32. hal-01629571

\section{HAL Id: hal-01629571 \\ https://hal.inria.fr/hal-01629571}

Submitted on 6 Nov 2017

HAL is a multi-disciplinary open access archive for the deposit and dissemination of scientific research documents, whether they are published or not. The documents may come from teaching and research institutions in France or abroad, or from public or private research centers.
L'archive ouverte pluridisciplinaire HAL, est destinée au dépôt et à la diffusion de documents scientifiques de niveau recherche, publiés ou non, émanant des établissements d'enseignement et de recherche français ou étrangers, des laboratoires publics ou privés. 


\title{
MAP Estimator for Target Tracking in Wireless Sensor Networks for Unknown Transmit Power
}

\author{
Slavisa Tomic ${ }^{4,5}$, Marko Beko ${ }^{1,3}$, Rui Dinis ${ }^{2,5}$, Milan Tuba ${ }^{6}$, Nebojsa Bacanin ${ }^{6}$ \\ ${ }^{1}$ CICANT-CIC.DIGITAL, Universidade Lusófona de Humanidades e Tecnologias, Lisbon, \\ Portugal \\ ${ }^{2}$ Instituto de Telecomunicações, Lisbon, Portugal \\ ${ }^{3}$ CTS, UNINOVA - Campus FCT/UNL, Caparica, Portugal \\ ${ }^{4}$ ISR/IST, LARSyS, Lisbon, Portugal \\ ${ }^{5}$ DEE/FCT, NOVA University of Lisbon, Caparica, Portugal \\ ${ }^{6}$ Faculty of Computer Science, John Naisbitt University, Belgrade, Serbia \\ \{s.tomic@campus.fc.unl.pt,mbeko@uninova.pt, rdinis@fct.unl.pt, tuba@ieee.org, \\ nbacanin@megatrend.edu.rs\}
}

\begin{abstract}
This paper addresses the target tracking problem, by extracting received signal strength (RSS) and angle of arrival (AoA) information from the received radio signal, in the case where the target transmit power is considered unknown. By combining the radio observations with prior knowledge given by the target transition state model, we apply the maximum a posteriori (MAP) criterion to the marginal posterior distribution function (PDF). However, the derived MAP estimator cannot be solved directly, so we tightly approximate it for small noise power. The target state estimate is then easily obtained at any time step by employing a recursive approach, typical for Bayesian methods. Our simulations confirm the effectiveness of the proposed algorithm, offering good estimation accuracy in all considered scenarios.
\end{abstract}

Keywords: Target tracking, maximum a posteriori (MAP) estimator, received signal strength (RSS), angle of arrival (AoA).

\section{Introduction}

\subsection{Motivation}

Locating a moving object in real-time has become an attractive topic in the academic and research community worldwide recently, owing to its great applicability potential in both military and commercial fields [1]-[5]. Taking advantage of the existing technologies, e.g., terrestrial radio frequency sources, to provide a solution to the realtime object localization problem is strongly encouraged, due to cost minimization. These include for example, extracting time of arrival, received signal strength (RSS), angle of arrival (AoA) information from the received radio signal, or a combination of them [5]-[11]. Which approach to use depends mainly on the available hardware. In this work, we employ combined RSS and AoA measurements, because nowadays, practically all devices can measure the RSS information, and the AoA information 
can be extracted from RSS measurements by using rotating a directional antenna and choosing the direction from which the highest RSS measurement is obtained [12],[13].

\subsection{Research Question}

Designing real-time localization algorithms is a very difficult task, because of numerous challenges that have to be taken into consideration, such as accuracy, execution time and limited energy resources to name a few. Therefore, the research question of this work can be formulated as:

How to design an efficient (highly accurate and computationally low complex) localization algorithm, robust to network topology and channel characteristics, applicable in real-time?

In order to address the research question, we have begun with the following hypothesis:

An efficient real-time localization algorithm can be developed by linearizing the considered non-linear measurement model and by following Bayesian approach, where prior knowledge is integrated with observations to enhance the estimation accuracy of an estimator.

\subsection{Related Work}

Works [6]-[11] investigated the classical target localization problem, where the proposed estimators were based on radio measurements only, and prior knowledge was disregarded. The authors in [2], [3] and [5] considered a problem of tracking of a moving target, and they combined the available radio measurements with prior knowledge given from the target state model. However, in [2], [3] and [5] only pure RSS-based target tracking problem was investigated. In [4], the target tracking problem which employs hybrid, RSS and AoA, measurements was considered. The authors in [4] proposed a Kalman filter (KF) and a particle filter (PF) to solve the tracking problem. Furthermore, they proposed a generalized pattern search method for estimating the path loss exponent (PLE) for each link in every time step.

\subsection{Contributions}

In this paper, we investigate the RSS-AoA-based target tracking problem for unknown target transmit power. This setting is of practical interest for low-cost systems in which testing and calibration is not the priority. Also, due to sensor's battery drain during time, the true value transmit power becomes not perfectly known over time. By integrating prior knowledge given by the target transition model and by employing the maximum a posteriori (MAP) approach, we propose a tight approximation of the MAP estimator. Based on a well-known recursive approach, typical for Bayesian methods, we develop an iterative algorithm which updates the mean and covariance of the target state in each time step. 


\section{Relationship to Smart Systems}

Smart systems embrace functions of sensing, actuation, and control with a goal of describing and analyzing an environment, and making decisions based on the available data in a predictive or adaptive manner. A good example of such a system is a wireless sensor network, composed of a number of scattered sensors that cooperate between themselves in order to respond in an attentive, adaptive and active way to the changes in the environment registered by sensors. However, in many applications, the information gathered by the sensors is meaningless if not correlated to accurate location of where the changes are occurring (e.g., a system might be set up to respond locally to changes in sensor data).

Therefore, determining accurate location of sensors is a very important task in forming a smart system. Moreover, accurate localization of objects and people in both indoor and outdoor environments enables new applications in emergency and commercial services that can improve safety and efficiency in everyday life (e.g., smart parking, monitoring of storage conditions and goods, assistance for elderly or people with disabilities) [14].

Another important example where target localization helps building a smart system is video surveillance. It has been used to monitor security sensitive areas such as banks, highways, borders, etc. Owing to rapid advances high-speed network infrastructure and computing power, as well as large capacity storage devices, multi sensor video surveillance systems have been developed recently. However, traditional video outputs that were controlled by human operators became overwhelming both for operators and storage devices, due to the increased number of cameras. Therefore, in order to filter out redundant information and increase the response time to forensic events motivated the development of smart video surveillance systems. Such systems require fast, reliable and robust algorithms for moving object detection, classification, tracking and activity analysis. [15]

\section{Problem Formulation}

Let $\boldsymbol{x}_{\mathrm{t}}=\left[x_{\mathrm{x}}, x_{\mathrm{y}}\right]^{T}$ and $\boldsymbol{a}_{\mathrm{i}}=\left[a_{\mathrm{ix}}, a_{\mathrm{iy}}\right]^{T}$, for $i=1_{s, \ldots} N$, denote the unknown location of a moving target at time instant ${ }^{t}$ andknown location of the ${ }^{i}$-th static anchor, respectively1. For simplicity, we assume a constant velocity target state transition model [1]-[5], [16],[17], i.e.,

$$
\theta_{t}=S \theta_{t-1}+r_{t}
$$

where $\theta_{t}=\left[x_{t}, v_{t}\right]^{T}$ represents the target state at time $t$ (described by its location and velocity, $v_{t}$, in a 2-dimensional plane) and $\boldsymbol{r}_{t}$ is the state process noise [1]-[5]. This process noise is assumed to be zero-mean Gaussian with a covariance matrix $\boldsymbol{Q}$, i.e., $\boldsymbol{r}_{\mathrm{t}} \sim \mathcal{N}(\mathbf{0}, \boldsymbol{Q})$. Covariance $\boldsymbol{Q}$ and state transition matrix, $\boldsymbol{S}$, are defined as

${ }^{1}$ For simplicity and without loss of generality, here we focus on 2-dimensional scenario. The generalization to a 3-dimensional scenario is straightforward. 


$$
\begin{gathered}
Q=q\left[\begin{array}{cccc}
\Delta^{\mathrm{a}} / 3 & 0 & \Delta^{2} / 2 & 0 \\
0 & \Delta^{\mathrm{a}} / 3 & 0 & \Delta^{2} / 2 \\
\Delta^{2} / 2 & 0 & \Delta & 0 \\
2 & \Delta^{2} / 2 & 0 & \Delta
\end{array}\right] \\
S=\left[\begin{array}{llll}
1 & 0 & \Delta & 0 \\
0 & 1 & 0 & \Delta \\
0 & 0 & 1 & 0 \\
0 & 0 & 0 & 1
\end{array}\right] x
\end{gathered}
$$

with $q$ and $\Delta$ denoting the state process noise intensity and the sampling interval between two consecutive time steps [1], [3], [16], [17].

At each time step, $t$, the target emits a signal to the anchors which then withdraw the RSS and AoA information from it. Thus, the observation model can be written as

$$
z_{t}=h\left(x_{t}\right)+n_{t} \text {, }
$$

where $z_{t}=\left[P_{t}^{T}, \phi_{t}^{T}\right]\left(z_{t} \in \mathbb{R}^{2 N}\right)$ is the observation vector composed of RSS, $\boldsymbol{P}_{\mathrm{t}}=\left[\boldsymbol{P}_{\mathrm{i}}^{(\mathrm{t})}\right]^{\underline{T}}$, and AoA, $\phi_{\mathrm{t}}=\left[\phi_{\mathrm{i}}^{(\mathrm{t})}\right]^{T}$, measurements at time instant $t$. The function $\boldsymbol{h}\left(\boldsymbol{x}_{\mathrm{t}}\right)$ in (2) is defined as $h_{\mathrm{i}}\left(\boldsymbol{x}_{\mathrm{t}}\right)=P_{0}-10 y \log _{10} \frac{\left\|x_{\mathrm{t}}-\boldsymbol{a}_{\mathrm{i}}\right\|}{d_{0}}$ for $i=1_{s \ldots s}, N$ [18], and $h_{\mathrm{i}}\left(x_{\mathrm{t}}\right)=\tan ^{-1}\left(\frac{x_{y}-a_{\mathrm{i}}}{x_{\mathrm{x}}-a_{\mathrm{ix}}}\right)$ for $i=N+1, \ldots, 2 N$, [19], where $P_{0}$ is the reference power at a distance $d_{0}$, and $\gamma$ is the PLE. The measurement noise is modeled as $n_{\mathrm{t}} \in \mathcal{N}(0, C)$, where the noise covariance is defined as $\boldsymbol{C}=\operatorname{diag}\left(\mid \sigma_{n_{\mathrm{i}^{\prime}}}^{2}, \sigma_{m_{\mathrm{i}}}^{2} J\right) \otimes \boldsymbol{I}_{4}$, with $\sigma_{n_{\mathrm{i}}}^{2}(\mathrm{~dB})$ and $\sigma_{m_{\mathrm{i}}}^{2}(\mathrm{rad})$ being the variances of the RSS and AoA measurement noise, respectively, $\boldsymbol{I}_{M}$ representing the identity matrix of size $M$ and symbol $\otimes$ denoting the Kronecker product. Often in practice, testing and calibration are not the priority in order to keep low network implementation costs; hence, the target transmit power, $P_{T}$, is often not calibrated, i.e., not known. Not knowing $P_{T}$ corresponds to not knowing $P_{0}$ in (2) [18], [20].

We employ (1) and (2) to build the marginal posterior probability distribution function (PDF), $p\left(\boldsymbol{\theta}_{t} \mid \boldsymbol{z}_{1-t}\right)$, from which we can quantify the confidence we have in the values of the state $\boldsymbol{\theta}_{t}$ given all the past measurements $\boldsymbol{z}_{1: t}$. From $p\left(\boldsymbol{\theta}_{t} \mid \boldsymbol{z}_{1-t}\right)$, we can obtain an estimate at any desired time step.

Below we show a recursive procedure, typical for Bayesian methods [1]-[5], for the evaluation of $p\left(\boldsymbol{\theta}_{t} \mid \boldsymbol{z}_{1: t}\right)$ at any time instant.

- Initialization: The marginal posterior PDF at $t=0$ is set to the prior PDF $p\left(\theta_{0}\right)$ of $\boldsymbol{\theta}_{0}$.

- Prediction: According to the state transition model (3), the predictive PDF of the state at $t$ is found as 


$$
p\left(\theta_{t} \mid z_{1: t-1}\right)=\int p\left(\theta_{t} \mid \theta_{t-1}\right) p\left(\theta_{t-1} \mid z_{1: t-1}\right) d \theta_{t-1} .
$$

- Update: By following the Bayes' rule [1], [13], we have

$$
p\left(\boldsymbol{\theta}_{t} \mid \boldsymbol{z}_{1: t}\right)=\frac{p\left(z_{t} \mid \boldsymbol{\theta}_{t}\right) p\left(\boldsymbol{\theta}_{t} \mid \mathbf{z}_{1: t-1}\right)}{\int p\left(z_{t} \mid \boldsymbol{\theta}_{t}\right) p\left(\boldsymbol{\theta}_{t} \mid \mathbf{z}_{1: t-1}\right) d \boldsymbol{\theta}_{t}} .
$$

It is worth noting that the denominator in (4) is just a normalizing constant. In general, the marginal PDF at $t-1$ cannot be calculated analytically, and the integral in (3) cannot be obtained analytically if the state model is non-linear. Therefore, some approximations are required in order to obtain $p\left(\boldsymbol{\theta}_{t} \mid z_{1-t}\right)$.

\section{The Proposed MAP Algorithm}

A state estimate, $\widehat{\theta}_{t \mid t}$, of $\boldsymbol{\theta}_{t}$ can be obtained from $p\left(\boldsymbol{\theta}_{t} \mid \boldsymbol{z}_{1: t}\right)$ by employing the MAP criteria [13], i.e., by maximizing the marginal posterior PDF

$$
\widehat{\boldsymbol{\theta}}_{\mathrm{t} \mid \mathrm{t}}=\underset{\boldsymbol{\theta}_{\mathrm{t}}}{\arg \max } p\left(\boldsymbol{\theta}_{\mathrm{t}} \mid \mathbf{z}_{1: \mathrm{t}}\right) \approx \underset{\boldsymbol{\theta}_{\mathrm{t}}}{\arg \max } p\left(\mathbf{z}_{\mathrm{t}} \mid \boldsymbol{\theta}_{\mathrm{t}}\right) p\left(\boldsymbol{\theta}_{\mathrm{t}} \mid \mathbf{z}_{1: t-1}\right) \mathrm{x}
$$

The problem in (5) resembles the maximum likelihood estimator, apart from the existence of the prior PDF. This problem is highly non-convex and its analytical solution cannot be obtained in general. Therefore, we approximate (5) by another estimator whose solution is readily available.

First, for small noise power we can write from the RSS and AoA model respectively

$$
\begin{gathered}
\lambda_{i}\left\|x_{\mathrm{t}}-\boldsymbol{a}_{\mathrm{i}}\right\| \approx \eta d_{0}, \text { for } i=1_{s, z} N_{x} \\
\boldsymbol{c}_{\mathrm{i}}^{T}\left(\boldsymbol{x}_{\mathrm{t}}-\boldsymbol{a}_{\mathrm{i}}\right) \approx 0, \text { for } i=N+1_{s, \ldots} 2 N_{x}
\end{gathered}
$$

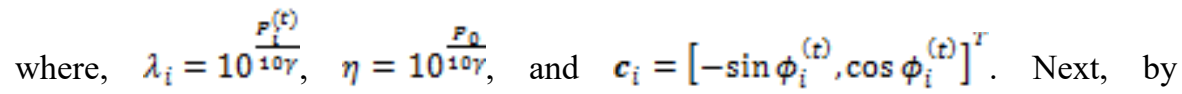
transforming from Cartesian to polar coordinates we express $\boldsymbol{x}_{\mathrm{t}}-\boldsymbol{a}_{\mathrm{i}}=r_{\mathrm{i}} \boldsymbol{u}_{\mathrm{i}}: r_{\mathrm{i}} \geq 0,\left\|\boldsymbol{u}_{\mathrm{i}}\right\|=1$, and make use of the available azimuth angle observations to define $\boldsymbol{u}_{\mathrm{i}}=\left[\cos \phi_{i}^{[\mathrm{t}]}, \sin \phi_{i}^{[\mathrm{t} t]}\right]$, and rewrite (6) as

$$
\lambda_{i} r_{\mathbb{1}} \approx \eta d_{0} .
$$

By multiplying the left hand side of the above equation by $\boldsymbol{u}_{\mathrm{i}}^{T} \boldsymbol{u}_{\mathrm{i}}$, we arrive at

$$
\lambda_{i} u_{i}^{T}\left(x_{t}-a_{i}\right) \approx \eta d_{0} .
$$


Hence, we can approximate the RSS and AoA measurement models by (8) and (7), respectively, which written in a vector form and applying the weighted least squares (WLS) criterion leads to

$$
\hat{y}_{t \mid t}=\underset{y_{t}=\left[x_{t}, n^{T}\right]^{T}}{\arg \min }\left\|W\left(A y_{t}-b\right)\right\|_{x}^{2}
$$

where $\boldsymbol{W}=I_{2} \otimes \operatorname{diag}(\boldsymbol{w}), \boldsymbol{w}=\left[\sqrt{w_{\mathrm{i}}}\right]^{T}$ and $w_{\mathrm{i}}=1-\frac{p_{i}^{(E)}}{\sum_{i=1}^{N} p_{i}^{(E)}}$

$$
\boldsymbol{A}=\left[\begin{array}{cc}
\vdots & \vdots \\
\lambda_{\mathrm{i}} \boldsymbol{u}_{\mathrm{i}}^{T} & d_{0} \\
\vdots & \vdots \\
\boldsymbol{c}_{\mathrm{i}}^{T} & 0 \\
\vdots & \vdots
\end{array}\right], \boldsymbol{b}=\left[\begin{array}{c}
\vdots \\
\lambda_{\mathrm{i}} \boldsymbol{u}_{\mathrm{i}}^{T} \boldsymbol{a}_{\mathrm{i}} \\
\vdots \\
\boldsymbol{c}_{\mathrm{i}}^{T} \boldsymbol{a}_{\mathrm{i}} \\
\vdots
\end{array}\right] .
$$

The solution of (9) is given by $\widehat{y}_{t \mid t}=\left(A^{T} W^{T} W A\right)^{-1}\left(A^{T} W^{T} b\right)$. Once we have an estimate of the target location, we can use it to find the maximum likelihood estimate of $P_{0}, \hat{P}_{0}$, from the RSS measurement model as

$$
\hat{P}_{0}=\frac{\sum_{i=1}^{W} P_{i}^{(t)}+10 y \log _{10} \frac{\left\|x_{t}-a_{i}\right\|}{\mathbb{d}_{0}}}{N} .
$$

At this point, we can benefit from this estimated value by using it to resume the estimation process as if $P_{0}$ is known, i.e., we can compute $\hat{\eta}=10^{\frac{P_{0}}{10_{Y}}}$ in order to get

$$
\widehat{\boldsymbol{A}}=\left[\begin{array}{ccc}
\vdots & \vdots & \vdots \\
\lambda_{i} \boldsymbol{u}_{\mathrm{i}}^{T} & 0 & 0 \\
\vdots & \vdots & \vdots \\
\boldsymbol{c}_{1}^{T} & 0 & 0 \\
\vdots & \vdots & \vdots
\end{array}\right], \widehat{\boldsymbol{b}}=\left[\begin{array}{c}
\vdots \\
\lambda_{\mathrm{i}} \boldsymbol{u}_{\mathrm{i}}^{T} \boldsymbol{a}_{\mathrm{i}}^{T}+\hat{\eta} d_{0} \\
\vdots \\
\boldsymbol{c}_{\mathrm{i}}^{T} \boldsymbol{a}_{\mathrm{i}}^{T} \\
\vdots
\end{array}\right] .
$$

As far as the prior knowledge part in (5) is concern, we can assume that $p\left(\boldsymbol{\theta}_{t-1} \mid z_{1: t-1}\right)$ has Gaussian distribution with mean $\widehat{\theta}_{t-1 \mid t-1}$ and covariance $\widehat{M}_{t-1 \mid t-1}[17]$. Then, according to (3), we obtain

$$
p\left(\theta_{t} \mid z_{1: t-1}\right) \approx \frac{1}{k} \exp \left\{-\frac{1}{2}\left(\theta_{t}-\widehat{\theta}_{t \mid t-1}\right)^{T} \widehat{M}_{t]-1}^{-1}\left(\theta_{t}-\widehat{\theta}_{t \mid t-1}\right)\right\}
$$

where $k$ is a constant, and $\widehat{\boldsymbol{\theta}}_{\mathrm{t} \mid \mathrm{t}-1}$ and $\widehat{M}_{\mathrm{t} \mid \mathrm{t}-1}$ are respectively the mean and covariance of the one-step predicted state, obtained through (1) as

$$
\begin{gathered}
\widehat{\theta}_{t \mid t-1}=S \widehat{\theta}_{t-1 \mid t-1}, \\
\widehat{M}_{t \mid t-1}=S M_{t-1 \mid t-1} S^{T}+Q .
\end{gathered}
$$

Therefore, (5) can be written as 


$$
\begin{aligned}
\widehat{\theta}_{t \mid t}=\underset{\theta_{t}}{\arg \min }\left(z_{t}-h\left(x_{t}\right)\right)^{T} C^{-1}\left(z_{t}-h\left(x_{t}\right)\right) \\
\quad+\left(\theta_{t}-\widehat{\theta}_{t \mid t-1}\right)^{T} \widehat{M}_{t]-1}^{-1}\left(\theta_{t}-\widehat{\theta}_{t \mid t-1}\right) .
\end{aligned}
$$

Similarly as in (9), the problem in (13) can be rewritten as

$$
\widehat{\theta}_{\mathrm{t} \mid \mathrm{t}}=\underset{\theta_{\mathrm{t}}}{\arg \min }\left\|\widetilde{W}\left(H \theta_{\mathrm{t}}-f\right)\right\|^{2},
$$

where

$$
H=\left[\widehat{A} ; \widehat{M}_{t \mid t-1}^{-\frac{1}{2}}\right], f=\left[\widehat{b}_{;} \widehat{M}_{t \mid t-1}^{-\frac{1}{2}} \widehat{\theta}_{t \mid t-1}\right], \quad \widetilde{W}=I_{2} \otimes \operatorname{diag}(\hat{w}),
$$

$\hat{\boldsymbol{w}}=\left[\hat{w}_{i}\right]^{T}, \hat{w}_{i}=1-\frac{d_{i}}{\sum_{i=1}^{N} \hat{d}_{i}}, \hat{d}_{i}=d_{0} 10^{\frac{p_{0}-P_{i}^{(t)}}{10}}$. The solution to (14) is given by $\widehat{\boldsymbol{\theta}}_{\mathrm{t} \mid \mathrm{t}}=\left(\boldsymbol{H}^{T} \boldsymbol{W}^{T} \boldsymbol{W}\right)^{-1}\left(\boldsymbol{H}^{T} \boldsymbol{W}^{T} \boldsymbol{f}\right)$.

The step by step proposed MAP algorithm for unknown $P_{T}$, is outlined in Algorithm 1.

Algorithm 1.The Proposed MAP Algorithm Description

$$
\begin{aligned}
& \text { Require: } z_{t} \text {, for } t=1_{s \ldots} T, Q, S \\
& 1: \text { Initialization: } \widehat{\boldsymbol{\theta}}_{0 \mid 0} \leftarrow(9), \widehat{M}_{0 \mid 0} \leftarrow \boldsymbol{I}_{4}, \hat{P}_{0} \leftarrow(10) \\
& \text { 2: for } t=1_{s \ldots} T \text { do } \\
& \text { 3: Prediction: } \\
& 4: \quad-\widehat{\theta}_{t \mid t-1} \leftarrow(12) \\
& \text { 5: } \quad-\widehat{M}_{t \mid t-1} \leftarrow(12) \\
& \text { 6: Update: } \\
& 7: \quad-\widehat{\theta}_{t \mid t} \leftarrow(14) \\
& \text { 8: } \quad-\widehat{M}_{\mathrm{t} \mid \mathrm{t}} \leftarrow \boldsymbol{I}_{4} \\
& \text { 8: } \quad-\hat{P}_{0} \leftarrow(10) \\
& \text { 10: end for }
\end{aligned}
$$

\section{Simulation Results}

This section validates the performance of the proposed algorithm through computer simulation. All simulations in this work were performed in MATLAB.

\subsection{Simulation Set-up}

Two scenarios, in which the target takes sharp maneuvers and a smoother one, are investigated. The state model (1) is used for the target state transition, and the radio 
measurements are acquired according to (2) at each time instant. Three anchors are fixed at $\left[[70,10]^{T}{ }_{s}[40,70]^{T}{ }_{v}[10,40]^{T}\right]$, and a sample is taken every $\Delta=1 \mathrm{~s}$ during $T=150 \mathrm{~s}$ trajectory duration in each Monte Carlo, $M_{c}=1000$, run. The reference distance is set to $d_{0}=1 \mathrm{~m}$, the reference power to $P_{0}=-10 \mathrm{dBm}$, and the PLE to $\gamma=3, \sigma_{n_{i}}=9 \mathrm{~dB}, \sigma_{m_{\mathrm{i}}}=4 \pi / 180 \mathrm{rad}$, and $q=2.5 \times 10^{-3} \mathrm{~m}^{2} / \mathrm{s}^{\mathrm{a}}$. The performance metric used here is the root mean square error (RMSE), defined as

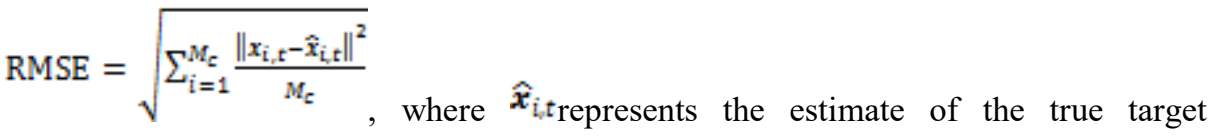
location, ${ }^{x_{i, t}}$, in the ${ }^{i}$-th Mc run at time instant ${ }^{t}$.

The performance of the proposed MAP algorithm is compared with a classical approach described by (9), which makes use of the radio measurements only and disregards the prior knowledge, labeled here as WLS.

\subsection{Results}

Fig. 1 illustrates the true target trajectory in the two considered scenarios as well as the estimated trajectories given by the considered approaches. In the first scenario, the initial target's location was set to $\left[21_{2} 20\right]^{T}$, whereas in the second scenario the starting point was set to $[35,15]^{T}$. We can see that the proposed MAP estimator performs considerably better in both scenarios than the WLS one. However, it seems like there is still room for further improvement of the proposed approach, since the results are not particularly smooth. This might be done by better utilization of the prior knowledge, i.e., in the update step of the state covariance matrix, and is left for future work. Fig. 2 illustrates the RMSE (m) versus $t$ (s) performance comparison of the considered approaches. From it, one can see that the proposed algorithm performs well in both scenarios, obtaining an average RMSE below $3 \mathrm{~m}$. Furthermore, the superiority of the Bayesian approach, which combines the observations with prior knowledge, over the classical one, which utilizes the observations exclusively, is clearly observed in every time step.

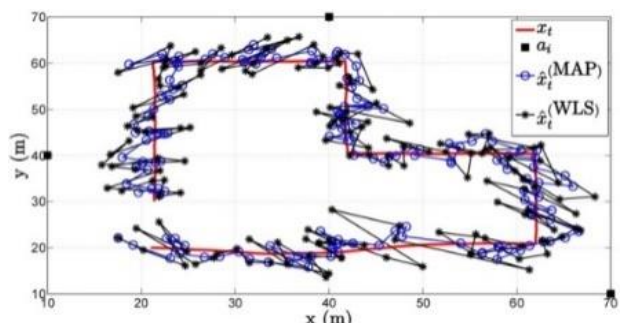

a)

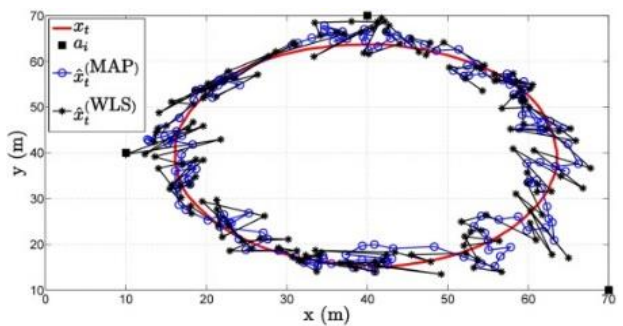

b)

Fig. 1. The true target trajectory in: a) the first and b) the second considered scenario and the estimated ones. 


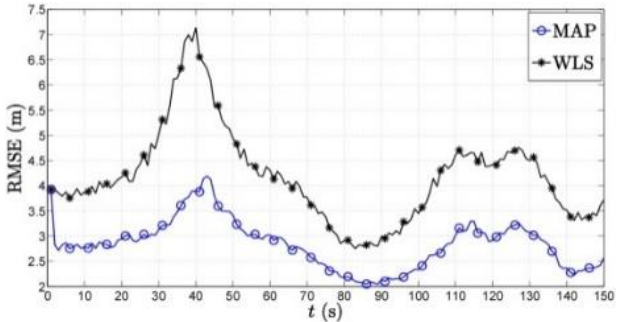

a)

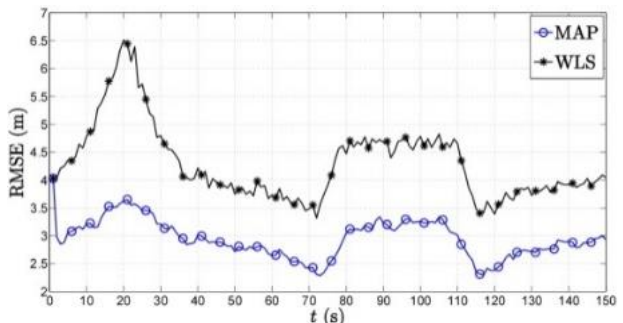

b)

Fig. 2. RMSE versus $t$ (s) comparison for a) the first and b) the second considered scenario.

\section{Conclusions}

In this work, we have investigated the target tracking problem which makes use of the combined RSS and AoA measurements, in which the target transmit power is considered not known. By resorting to Bayesian approach and relying on the MAP criterion, we proposed a tracking algorithm, which efficiently solves the target tracking in all considered scenarios and significantly outperforms the considered classical approach in every time instant. Although the proposed algorithm offers excellent estimation accuracy, it seems that there is still room for further improvement, which will be the topic of our future work.

This work is a part of our ongoing research, and we have validated the performance of our algorithms by means of simulations only. As a part of our future work, we plan to validate their performance using real measurements from [12] and [13].

Acknowledgments. This work was partially supported by Fundação para a Ciência e a Tecnologia under Project PEst-OE/EEI/UI0066/2014 (UNINOVA), and Project UID/EEA/50008/2013 (Instituto de Telecomunicações), Program Investigador FCT under Grant IF/00325/2015 and Grant SFRH/BD/91126/2012.

\section{References}

1. Dardari, D., Closas, P., Djuric, P. M.: Indoor Tracking: Theory, Methods, and Technologies. In IEEE Trans. Vehic. Technol., vol. 64, no. 4, pp. 1263--1278 (2016).

2. Beaudeau, J. P., Bugallo, M. F., Djuric, P. M.: RSSI-based Multitarget Tracking by Cooperative Agents Using Fusion of Cross-target Information. In IEEE Trans. Sign. Process., vol. 63, no. 19, pp. 5033--5044 (2015).

3. Masazade, E., Niu, R., Varshney, P. K.: Dynamic Bit Allocation for Object Tracking in Wireless Sensor Networks. In IEEE Trans. Sign. Process., vol. 60, no. 10, pp. 5048-5063 (2012). 
4. Khan, M. W., Kemp, A. H., Salman, N., Mihaylova, L. S.: Tracking of Wireless Mobile Nodes in the Presence of Unknown Path-loss Characteristics. In Fusion, Washington DC, USA, pp. 104-111 (2015).

5. Khan, M. W., Salman, N., Ali, A., Khan, A. M., H. Kemp, A.: A Comparative Study of Target Tracking With Kalman Filter, Extended Kalman Filter and Particle Filter Using Received Signal Strength Measurements. In 11th IEEE International Conference on Emerging Technologies (ICET), Peshawar, Pakistan, pp. 1--6 (2015).

6. Tomic, S., Beko, M., Dinis, R.: Distributed RSS-Based Localization in Wireless Sensor Networks Based on Second-Order Cone Programming. In Sensors, vol. 14, no. 10, pp. $18410-18432$ (2014)

7. Tomic, S., Beko, M., Dinis, R.: RSS-based Localization in Wireless Sensor Networks Using Convex Relaxation: Noncooperative and Cooperative Schemes. In IEEE Trans. Veh. Technol., vol. 64, no. 5, pp. 2037-2050 (2015)

8. Tomic, S., Beko, M., Dinis, R.: 3-D Target Localization in Wireless Sensor Network Using RSS and AoA Measurement. In IEEE Trans. Vehic. Technol., vol. PP, no. 99, pp. 1, Jul. 2016.

9. Tomic, S., Beko, M., Dinis, R.: Distributed RSS-AoA Based Localization with Unknown Transmit Powers. In IEEE Wirel. Commun. Letters, vol. 5, no. 4, pp. $392--395$ (2016).

10.Tomic, S., Beko, M., Dinis, R., Montezuma, P.: Distributed Algorithm for Target Localization in Wireless Sensor Networks Using RSS and AoA Measurements. In Pervasive and Mobile Computing, vol. PP, no. 99, pp. 1-1 (2016).

11.Tomic, S., Beko,M., Dinis,R., Montezuma,P.: A Closed-form Solution for RSS/AoA Target Localization by Spherical Coordinates Conversion. In IEEE Wirel. Commun. Letters, vol. PP, no. 99, pp. 1-1 (2016).

12.Niculescu D., Nath, B.: Vor Base Stations for Indoor 802.11 Positioning", In the 10thAnnual International Conference on Mobile Computing and Networking(ACM MobiCom), Philadelphia, PA, USA, pp. 58-69 (2004).

13.Elnahrawy, E., Francisco, J. A., Martin, R. P.: Adding Angle of Arrival Modality to Basic RSS Location Management Techniques", In the 2nd International Symposium on Wireless Pervasive Computing (ISWPC), San Juan, PR, USA, pp. 1-6 (2007).

14.Yang, J., Portilla, J., Riesgo, T.: Smart Parking Service based on Wireless Sensor Networks. In the 38th Annual Conference on IEEE Industrial Electronics Society (IECON), Montreal, QC, Canada, pp. 6029--6034 (2012).

15.Y. Dedeoglu: Moving Object Detection, Tracking and Classification for Smart Video Surveillance. Master Thesis, Bilkent University, Ankara, Turkey (2004).

16.Kay. S. M.: Fundamentals of Statistical Signal Processing: Estimation Theory. PrenticeHall, Upper Saddle River, NJ, USA (1993).

17.Wang, G., Li, Y., Jin, M.: On MAP-based Target Tracking Using Range-only Measurements. In 8th International Conference on Communications and Networking in China (CHINACOM), Guilin, China, pp. 1-6 (2013).

18.Rappaport, T. S.: Wireless Communications: Principles and Practice. Prentice-Hall, Upper Saddle River, NJ, USA (1996).

19.Yu, K.: 3-D Localization Error Analysis in Wireless Networks. In IEEE Trans. Wirel. Commun., vol. 6, no. 10, pp. 3473-3481 (2007).

20.Patwari, N., Ash, J. N., Kyperountas, S., Hero III, A. O., Moses, R. L., Correal, N. S.: Locating the Nodes: Cooperative Localization in Wireless Sensor Networks, IEEE Sign. Process.Mag., vol. 22, no. 4, pp. 54--69 (2005). 